those with the disorder voiced the view strongly that they found the term both stigmatising and embarrassing. Clinicians and those affected generally considered that the term incorrectly implies the condition is gastrointestinal rather than neurological and, as a consequence, those professionals who should be interested in it are often not.

All those involved considered that the term pain should remain. Most clinicians were initially sceptical about qualifying this with words such as severe, extreme or excruciating, often feeling that the use of these would be frightening, especially at diagnosis. Those affected and their families disagreed, arguing that as the condition is associated with episodes of pain that seem to be as severe as anything experienced by man, this must be reflected in the name. Indicating the severity of the pain will emphasise to clinicians the importance of recognising the disorder, particularly as partially effective treatments are available. Finally, after much debate, most considered that the best term to describe the pain was "extreme".

Clinicians and scientists were of the opinion that as we now understand what causes the condition and know its clinical features and prognosis, the term syndrome is no longer appropriate and that either disease or disorder should be substituted. Those affected and their families preferred the term disorder as being less stigmatising.

Most initially wanted to retain the term familial. There was, however, eventually an agreement to drop it, partly because a fiveword name was considered excessively cumbersome and partly in acknowledgment that sporadic cases also occur.

Whether other features of the disorder, such as the harlequin colour changes and the non-epileptic tonic seizures, should be reflected in the name was discussed. It was, however, felt that some of these features, such as colour changes, although interesting, have a minor effect on those affected, whereas others, such as seizures, are not universal. Clinicians strongly thought that the episodic nature of the attacks should be reflected in the name. The term paroxysmal was preferred to seizure as this was less likely to lead to confusion with epilepsy.

The name that received most support was paroxysmal extreme pain disorder (PEPD). Given that this name emerged from a democratic process including, as far as we know, most professionals interested in the condition and affected patients and their families from around the world, we consider that henceforth the name "paroxysmal extreme pain disorder" should be used in preference to the obsolete name familial rectal pain (syndrome).

\section{R Fertleman}

Department of Paediatrics and Child Health, Royal Free and University College London, London, UK

C D Ferrie Department of Paediatric Neurology, Leeds General
Infirmary, Leeds, UK

Correspondence to: Caroline R Fertleman Department of Paediatrics and Child Health, Royal Free and University College London, 5 University Street, London WC1E 6JJ, UK; c.fertleman@ucl.ac.uk

The worldwide consortium has consulted and includes the following in alphabetical order: Jean Aicardi, Mark Baker, Orvar Eeg-Olofsson, Frances Elmslie, Martin Kirkpatrick, Ingemar Malmros, Sarah Moffatt Keith Parker, Melanie Pollitzer, Michele Rees, Mary

Rossiter, Eliane Roulet-Perez, Romaine Schubert, John Stephenson, Herve Testard and Virginia Wong. doi: 10.1136/jnnp.2006.089664

Competing interests: None declared.

\section{References}

1 Hayden R, Grossman M. Rectal, ocular, and submaxillary pain; a familial autonomic disorder related to proctalgia fugax: report of a family. Am J Dis Child 1959;97:479-82.

2 Dugan RE. Familial rectal pain. Lancet $1972 ; 1: 854$.

3 Fertleman CR, Baker MD, Parker KA, et al. SCN9A mutations in paroxysmal extreme pain disorder: allelic variants underlie distinct channel defects and phenotypes. Neuron (in press).

\section{Email triage for new neurological outpatient referrals: what the customers think}

When patients with neurological symptoms are referred to a neurologist by their general practitioner (GP), the neurologist acquires two customers-the patient and the GP. If the neurologist plans to introduce a new or changed service it would be considered good practice to seek the opinion of the people using the service-the customers. Such an approach is commonplace in other walks of life, but is rarely used in medicine.

We used this approach when considering changing from a letter-based referral system for new referrals to one based on email. Email can be used to triage referred patients into those who need to be seen in a clinic and those who can be managed without entering the clinic system, either by investigations or by simply offering advice. This approach has been used successfully for general medical patients in Finland ${ }^{1}$ and we have shown that in neurological practice this novel method is feasible, safe, sustainable and saves neurolo-

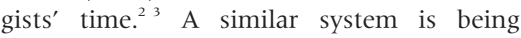
introduced within parts of the UK National Health Service for a variety of specialties under the title of Integrated Clinical Assessment and Treatment Services. ${ }^{4}$ The email service provides a response to the GP within $48 \mathrm{~h}$, but if the referral is dealt with by simply providing advice or by arranging investigations, the patient will not be seen in person by the neurologist. When we presented our results at neurological meetings, one objection raised against the email system was that patients and their GPs would be unhappy that a referred patient may not see a neurologist in person. To test this hypothesis we carried out a customer survey.

\section{Methods}

The study was carried out in two different parts of the UK, general neurology clinics throughout Northern Ireland and waiting list initiative clinics in Pinderfields Hospital, Wakefield, Yorkshire.

Patients were new referrals from GPs who had been referred conventionally by letter and whose degree of urgency had been graded by the neurologist as routine. They had generally been waiting between 3 and 6 months for their appointment.

Their opinion was obtained while they were waiting to be seen by the neurologist. The approval of the ethical committee was not required.

Patients were asked to choose between the two possible referral methods that were presented to them on a single sheet of paper. The sheet was given to them by the clinic nurse or receptionist, with a short written explanation, and the completed sheet was collected from them before they saw the neurologist, who was unaware of the results when seeing the patient.

GPs were chosen in Northern Ireland by geographical spread, excluding County Fermanagh, where the email system had already been introduced. They were contacted by telephone by one of the authors (CD) and the questionnaire was filled in over the telephone.

GPs in Wakefield were chosen from hospital referrers. They were contacted first by an explanatory letter enclosing the questionnaire and if this was not returned, one of the authors (LL) followed up by telephone.

\section{Results}

Overall, $80 \%$ of patients in Northern Ireland $(n=100)$ and $91 \%$ of those in Yorkshire $(n=100)$ preferred the email system. GPs also preferred the email system, although less enthusiastically so- $80 \%$ in Northern Ireland $(\mathrm{n}=50)$ and $51 \%$ in Yorkshire $(\mathrm{n}=82)$.

\section{Discussion}

Most new patients and GPs in two parts of the UK would prefer an email triage system compared with the current system of hospital referral. We carried out this study on patients who were within the system but had not yet completed it and who therefore had good knowledge of what the conventional system included. All the GPs had first-hand knowledge of referral of patients to their local neurologist. The wording of the questions was direct but encapsulated the implicit trade-off between an early opinion with the possibility of not being seen by a specialist against a delayed opinion with the guarantee of being seen by a specialist.

We do not find these results surprising. The existing system of referral is impersonal and inefficient. Hospital referral letters are usually unstructured and many are still hand written and often difficult to read. An email with a proforma encapsulates what the GP wants to gain from the consultation and the rapid response from the neurologist brings a degree of personal interaction that is completely lacking in the conventional system. A competent neurologist, from a description by a competent GP, can easily diagnose many neurological symptoms of patients. Patients can then either be reassured without being seen or have appropriate investigations carried out without entering the hospital system. A follow-up of more than 150 patients by this system has shown that, when carried out by an experienced neurologist, this practice is safe and saves neurologists' time. ${ }^{3}$ Benefits accrue to both patients and their GPs and also to neurologists who will see patients in the clinic more relevant to their skill and experience. A wider adoption of this system requires neurologists and GPs to change their practice and such changes are known to be difficult. Whatever the arguments proposed against these changes, a lack of customer readiness should not be one of them.

V Patterson

Centre for Online Health, Brisbane, Australia

C Donaghy

Royal Victoria Hospital, Belfast, UK 
L Loizou

Pinderfields Hospital, Wakefield, UK

Correspondence to: Dr Victor Patterson, Teleneurology, 1st Floor Education Centre, Royal Victoria Hospital, Belfast BT12 6BA, UK: tele.neuro@royalhospital.n-i.nhs.uk

doi: 10.1136/jnnp.2006.092098

Competing interests: None.

\section{References}

1 Harno KS. Telemedicine in managing demand for secondary-care services. J Telemed Telecare 1999;5: 189-92.

2 Patterson V, Humphreys J, Chua R. Email triage of new neurological outpatient referrals from general practice. I Neurol Neurosurg Psychiatry 2004;75:617-20.

3 Patterson V, Humphreys J, Chua R. Teleneurology by email. J Telemed Telecare 2003;9/Suppl 2):42-3.

4 Baxter C. Major investment in outpatient reform. www.nics.gov.uk/press/hss/060116f-hss.htm 4 (accessed 28 Feb 2006).

\section{Now dear, I have a headache! Immediate improvement of cluster headaches after sexual activity}

The precise pathogenesis of cluster headaches is unknown, but a hypothalamic generator has been postulated as the cause of the disorder. ${ }^{1}$ In two patients with typical cluster headaches, sexual activity alleviated the episodes. This association may shed some light on the pathogenesis and treatment of this incapacitating disorder.

Patient 1: A 61-year-old, previously healthy man presented with excruciating, left-sided orbital headaches appearing in bouts that lasted for 10 weeks. The pain occurred nightly at around 22:00 $\mathrm{h}$, and was associated with ipsilateral ptosis, lacrimation and rhinorrhoea. Neurological examination and brain imaging were normal and he was diagnosed with cluster headaches, according to International Headache Society (IHS) criteria. ${ }^{2}$ The headaches always lasted for 90-150 min, except for instances when the patient had sexual intercourse, which at the point of orgasm resulted in instant dramatic improvement in the pain, with complete relief always being achieved within several minutes and no recurrence until the next evening. Orgasm occurred on six occasions between 5-30 min after the onset of the episode and termination of the episode followed in all instances. Prophylactic treatment with verapamil was initiated and he was advised to try oxygen inhalation. Adherence to this regimen abolished the current cluster of headache episodes.

Patient 2: A 47-year-old previously healthy man had episodes of headache appearing in clusters since his teens. Episodes consisted of unilateral orbital pain with lacrimation and rhinorrhoea that lasted for 30-60 min, with a frequency of 1-3 episodes daily for several weeks. His headaches fitted IHS criteria for cluster headache. ${ }^{2}$ For two decades, he was treated with triptans and prophylactic indomethacin, and rarely had headaches. Cessation of the treatment was followed by relapse. More than a decade after discontinuing treatment, he consulted a neurologist and reported that over the years he had learnt that sexual intercourse and masturbation were linked with instant relief from the headache at the point of orgasm. Neurological examination and brain imaging were normal. Prophylactic treatment with indomethacin was reinstituted, with no episodes reported over the subsequent 2 years of follow-up.

\section{Discussion}

The link between sexual activity and cluster headaches has been discussed before Patients with cluster headaches (but not healthy controls) respond to testosterone administration by increasing their sexua behaviour, which may suggest a derangement of central nervous system processes associated with libido. ${ }^{3}$ The case of a patient who had cluster headaches which disappeared during the period of involvement in a sexual relationship but subsequently relapsed after the termination of the relationship, has been described. ${ }^{4}$ Although cluster headaches have been reported to be triggered by intercourse, ${ }^{4}$ in our patients the headache was well established before the initiation of sexual activity.

Our observation is novel in that it documents the termination of individual cluster headache episodes by orgasm and could relate to one or more of the processes underlying the pathophysiology of cluster headaches. According to the "gate theory", sexual activity may activate inhibitory painmodulating circuits. ${ }^{5}$ This phenomenon occurs in many situations in which there is a survival value in not "giving-in" to pain and may also be responsible for the placebo effect. Related to this process is the possibility that reduction in headache could be related to endorphin excretion, which occurs after sexual arousal and orgasm.

While these pain-reducing processes could equally apply to any nociceptive experience, more specific neuroanatomical relationships between sexual activity and cluster headaches exist. The episodic nature of cluster headaches suggests the involvement of a central impulse generator or oscillator, and a biological clock within the hypothalamus has been implicated. ${ }^{1}$ Positron emission tomography scanning showed an intense activation of the posterior hypothalamus during an episode, and refractory patients respond to hypothalamic deep brain stimulation. ${ }^{7}$ Intriguingly, orgasm is accompanied by intense hypothalamic activation. ${ }^{6}$ Thus, it can be postulated that orgasm terminates cluster headaches by modulating hypothalamic circuits in a manner similar to that which occurs in deep brain stimulation.

Patient 2, although satisfying IHS criteria for cluster headaches, is notable for his response to indomethacin. Although cluster headaches have been reported to respond to indomethacin, ${ }^{8}$ this feature usually suggests paroxysmal hemicrania ${ }^{2}$ and raises the possibility that this phenomenon may be relevant to the trigeminal autonomic cephalgias (TAC) as a group, all of which are associated with the posterior hypothalamus.'

The sympathetic nervous system is believed to be associated with TAC in a passive manner ${ }^{1}$; however, in some cases, there is evidence of sympathetic dysfunction before the onset of TAC, ${ }^{10}$ suggesting that sympathetic underactivity may underlie TAC pathogenesis. If TAC pain is dependent on low sympathetic tone, theoretically the episode could be reversed by the increase in sympathetic activity accompanying sexual activity and, specifically, orgasm.

Patients with TAC may be reluctant or unable to engage in intercourse during an episode or disinclined to volunteer such information. Given that other sufferers may potentially benefit from this phenomenon, and as it has relevance to the pathogenesis of TAC, this observation should be verified in a large cohort of patients.

M Gotkine, I Steiner, I Biran

Department of Neurology, Hadassah University Hospital and the Hebrew University-Hadassah Medical School, Jerusalem, Israel

Correspondence to: M Gotkine, Department of Neurology, Hadassah University Hospital, Box 12000, Jerusalem 91 120, Israel; marcgotkine@gmail.

Informed consent has been obtained for publication of the patient details in this article.

doi: 10.1136/innp.2006.092643

Competing interests: None declared.

\section{References}

1 May A, Bahra A, Buchel C, et al. Hypothalamic activation in cluster headache attacks. Lancet 1998;352:275-8.

2 Silberstein CD, Olesen J, Bousser MG, et al. The International Classification of Headache Disorders. 2nd edn. Cephalalgia 2004;24(Suppl 1):9-160.

3 Nicolodi M, Sicuteri F, Poggioni M. Hypothalamic modulation of nociception and reproduction in cluster headache. II. Testosterone-induced increase of sexual activity in males with cluster headache. Cephalalgia 1993;13:258-60.

4 Maliszewski M, Diamond S, Freitag FG. Sexual headaches occurring in cluster headache patients. Clin J Pain 1989:5:45-7.

5 Sluka KA, Walsh D. Transcutaneous electrical nerve stimulation: basic science mechanisms and clinical effectiveness. J Pain 2003:4:109-21.

6 Meston CM, Frohlich PF. The neurobiology of sexual function. Arch Gen Psychiatry 2000;57:1012-30

7 Leone M, Franzini A, Broggi G, et al. Long-term follow-up of bilateral hypothalamic stimulation for intractable cluster headache. Brain 2004;127(Pt 10):2259-64

8 Buzzi MG, Formisano R. A patient with cluster headache responsive to indomethacin: any relationship with chronic paroxysmal hemicrania? Cephalalgia 2003;23:401-4.

9 Matharu MS, Cohen AS, Frackowiak RS, et al. Posterior hypothalamic activation in paroxysmal hemicrania. Ann Neurol 2006:59:535-45.

10 Havelius U. A Horner-like syndrome and cluster headache. What comes first? Acta Ophthalmol Scand $2001 ; 79: 374-5$

\section{Novel Olig 1-coding variants and susceptibility to multiple sclerosis}

Oligl is a basic helix-loop-helix (bHLH) transcription factor expressed in cells of the oligodendrocyte lineage in the nervous sys tem. Its role during normal development has not yet been fully resolved, but it is known that in adult life the protein is crucial in the process of remyelination after injury. ${ }^{1-3}$ Olig 1 translocates from the cytoplasm to the nucleus in early remyelinating lesions in rodent models of demyelinating disease as well as in oligodendrocyte precursor cells at the edge of multiple sclerosis lesions. ${ }^{1}$ Olig specifically regulates the expression of the 